\title{
Traumatismes fermés des bourses : stratégie de prise en charge
}

\author{
Hammadi FAKHFAKH, Kamel CHABCHOUB, Abdelkader BOUHLEL, Hafedh KETATA, Hatem \\ ALLOUCH, Ali BAHLOUL, Mohamed Nabil MHIRI
}

Service d'urologie, CHU Habib BOURGUIBA, Sfax, Tunisie

RESUME

Introduction : Les traumatismes fermés des bourses constituent une urgence médico-chirurgicale ; et sont de plus en plus fréquents. La nécessité d'explorer chirurgicalement en urgence ces traumatismes reste une question controversée.

Le but de ce travail est d'analyser les principaux facteurs étiologiques, de détailler les différents aspects cliniques et thérapeutiques de ces traumatismes, de mettre en exergue le rôle apporté par l'échographie scrotale et d'établir les éléments de pronostic.

Patients et Méthode : Cent trente traumatismes fermés des bourses ont été pris en charge entre 1993 et 2004. En l'absence de critère de gravité clinique et échographique (hématocèle, volumineux hématome intra testiculaire, rupture de l'albuginée) un traitement médical a été instauré. En cas de suspicion de lésions graves du testicule, une exploration chirurgicale a été réalisée.

L'échographie scrotale a été pratiquée chez 68 patients dont $\mathbf{4 6}$ ont été explorés chirurgicalement. Ainsi, nous avons apprécié la sensibilité et la spécificité de l'échographie en confrontant les données de celle-ci aux résultats de l'exploration peropératoire. La morbidité immédiate et les séquelles à distance ont été analysées en fonction du traitement proposé.

Résultats : L'âge moyen de nos patients était de 24 ans (4 à 73 ans). La symptomatologie clinique était dominée par la douleur $(73,8 \%)$ et l'augmentation de volume du scrotum $(89,2 \%)$. La sensibilité de l'échographie a été de $34,7 \%$ pour les fractures testiculaires, de $100 \%$ pour les contusions testiculaires, de $33,3 \%$ pour l'hématome intra testiculaire et de $76,9 \%$ pour l'hématocèle. Le traitement médical et le traitement chirurgical ont été instaurés respectivement dans $29,2 \%$ et $70,7 \%$ des cas. Après un recul moyen de 8 mois, les complications les plus fréquentes étaient les douleurs résiduelles (18\%) et l'atrophie testiculaire $(5,5 \%)$

Conclusion : En l'absence de signes de gravité, un traitement médical reste justifié avec une surveillance régulière, mais en cas de doutes cliniques ou échographiques, une exploration chirurgicale doit être réalisée dans de brefs délais.

Mots clés : traumatisme, scrotum, testicule, échographie

\section{INTRODUCTION}

Les traumatismes fermés des bourses constituent une urgence médico-chirurgicale et sont de plus en plus fréquents. Ils touchent souvent l'adulte jeune et l'adolescent. Ces traumatismes peuvent s'accompagner de difficultés diagnostiques pour la réalisation d'un bilan lésionnel précis et complet, et posent un dilemme dans le choix entre un traitement médical conservateur et un traitement chirurgical qui risque d'être radical. De même l'importance des complications et des séquelles, qui peuvent s'en suivre, justifie une prise en charge rapide en milieu urologique

Le but de ce travail est d'analyser les principaux facteurs étiologiques, de détailler les différents aspects cliniques et thérapeutiques de ces traumatismes, de mettre en exergue le rôle apporté par l'échographie scrotale et d'établir les éléments de pronostic afin de préserver les testicules et leurs fonctions.

Correspondance :

Dr Hammadi FAKHFAKH - Service d'urologie, CHU Habib Bourguiba, Sfax,Tunisie - Tel 0021698639784 -

Email h.fakhfakh@laposte.net 


\section{MATERIEL ET METHODES}

Notre travail est basé sur une étude rétrospective à propos de 130 observations colligées sur une période de 12 ans (Janvier 1993 à Décembre 2004). Tous les patients ont consulté en urgence pour un traumatisme scrotal fermé par choc direct ou suite à une chute en califourchon. Ils ont été hospitalisés et examinés par un urologue.

Une échographie scrotale a été pratiquée chez 68 patients dont 46 ont été explorés chirurgicalement. Ainsi, nous avons pu apprécier la sensibilité et la spécificité de l'échographie en confrontant les données de celle-ci aux résultats de l'exploration per-opératoire.

Enfin nous rapportons pour l'ensemble de nos patients, les résultats des différentes données épidémiologiques, cliniques, para-cliniques, thérapeutiques et évolutives.

\section{RESULTATS}

L'âge moyen de nos patients au moment du traumatisme était de 24 ans avec des extrêmes allant de 4 ans à 73 ans et un pic de fréquence entre 10 et 29 ans $(72,3 \%$ des cas).

Le délai moyen entre la consultation et le traumatisme initial était de 18,5 jours avec des extrêmes allant de quelques heures à 2 ans. Soixante quinze patients $(57,7 \%)$ ont consulté au cours des 24 premières heures, tandis que 30 patients $(23 \%)$ ont consulté au delà du quatrième jour après leur traumatisme. Le testicule gauche a été affecté dans 61 cas $(46,9 \%)$, et le testicule droit dans 56 cas $(43 \%)$. Le traumatisme était bilatéral dans 13 cas $(10 \%)$.

Les circonstances étiologiques les plus fréquemment retrouvées à l'interrogatoire, étaient les agressions ou les rixes ( 39 cas), les accidents domestiques ( 24 cas), les accidents de la voie publique (18 cas), les accidents sportifs (18 cas) et les accidents de travail (13 cas). L'accident était en rapport avec un choc direct (74 cas) ou une chute à califourchon (21 cas).

Dans 11 cas le traumatisme scrotal était associé à d'autres lésions à type de traumatisme crânien (4 cas), de fracture des os long (5 cas), de fracture du bassin ( 2 cas) dont une compliquée de rupture de l'urètre postérieur, tous ces patients étaient victimes d'un accident de la voie publique.

La symptomatologie clinique était dominée par la douleur scrotale et l'augmentation du volume de la bourse, observées respectivement, chez 96 patients $(73,8 \%)$ et 116 patients $(89,2 \%)$. Un hématome scrotal était présent dans 44 cas $(33,8 \%)$. La palpation des bourses était tolérable chez 83 patients $(63,8 \%)$. Le diagnostic clinique d'une hématocèle n'a pu être fait que dans 3 cas. Ainsi la sensibilité de l'examen clinique pour la détection d'une hématocèle est de 5,6\%. Une luxation testiculaire était retrouvée chez un seul patient au niveau du canal inguinal, alors que dans 7 cas le testicule était ascensionné. La palpation des annexes a permis de découvrir 5 cas de tuméfaction épididymaire et un cas de tuméfaction en regard du cordon.

L'échographie scrotale a été réalisée chez 68 patients (Tableau 1). Parmi eux 46 ont eu une exploration chirurgicale. Nous avons pu apprécier la sensibilité et la spécificité de l'échographie en confrontant les données de celle-ci aux résultats de l'exploration peropératoire (Tableau 2). Ainsi, pour les fractures testiculaires

Tableau 1: Résultats échographiques (68 patients).

\begin{tabular}{|c|c|c|c|}
\hline & $\begin{array}{c}\text { Types } \\
\text { de lésions }\end{array}$ & $\begin{array}{l}\text { Nombre } \\
\text { de cas }\end{array}$ & Pourcentages \\
\hline & $\begin{array}{l}\text { Contusion } \\
\text { testiculaire }\end{array}$ & 23 & 33,8 \\
\hline \multirow[t]{2}{*}{$\begin{array}{l}\text { Lésions } \\
\text { testiculaires }\end{array}$} & $\begin{array}{l}\text { Hématome } \\
\text { intra-testiculaire }\end{array}$ & 11 & 16,1 \\
\hline & Fracture testiculaire & 8 & 11,7 \\
\hline \multicolumn{2}{|l|}{ Hématocèle } & 26 & 38,2 \\
\hline \multicolumn{2}{|c|}{ Lésions épididymaires } & 14 & 20,5 \\
\hline $\begin{array}{l}\text { Lésions des } \\
\text { enveloppes }\end{array}$ & Hématome pariétal & 11 & 16,1 \\
\hline testiculaires $\mathrm{E}$ & paississement pariétal & 4 & 5,8 \\
\hline
\end{tabular}

Tableau 2 : Corrélations anatomo-radiologiques (46 patients).

\begin{tabular}{lcccccc}
\hline Type de lésion & FP & FN & VP & VN & SSTE & SPTE \\
Contusion testiculaire & 15 & 0 & 3 & 28 & 100 & 65,1 \\
Fracture testiculaire & 0 & 15 & 8 & 21 & 34,7 & 100 \\
Hématome intra-testiculaire & 6 & 2 & 1 & 37 & 33,3 & 86 \\
Hématocèle & 1 & 6 & 20 & 19 & 76,9 & 95,2 \\
Lésions des annexes & 9 & 12 & 3 & 22 & 20 & 70,9 \\
\hline
\end{tabular}

SSTE: sensibilité ; SPTE: spécificité ; FP : faux positif ; FN : faux négatif ; VP : vrai positif ; VN : vrai négatif 
l'échographie avait une sensibilité de $34,7 \%$ avec une spécificité de $100 \%$.

Par ailleurs, la sensibilité de l'échographie était de 100\% pour les contusions testiculaires, de $33,3 \%$ pour l'hématome intra testiculaire, de $76,9 \%$ pour l'hématocèle, et de $15,8 \%$ pour la détection des lésions des annexes.

Le traitement médical, anti-inflammatoire, antalgique, repos et suspensoir scrotal, a été instauré pour une durée moyenne de 10 à 15 jours dans 38 cas $(29,2 \%)$. L'évolution sous traitement médical a été défavorable chez 13 patients $(34,2 \%)$. En effet, après un suivi moyen de 22 jours, celle ci a été marqué par la persistance de la symptomatologie, la réapparition de la douleur et ou l'augmentation du volume scrotal.

Le traitement chirurgical a été réalisé chez 92 patients $(70,7 \%)$, d'emblée devant toute suspicion de fracture testiculaire ou secondairement après échec d'un traitement médical (13 cas). L'exploration chirurgicale a été pratiquée dans un délai moyen de 72 heures chez 73 patients $(69,5 \%)$. Par contre dans 32 cas l'exploration a été plus tardive. La voie d'abord a été principalement scrotale $(95,2 \%$ des cas $)$.

Le traitement chirurgical était variable en fonction des lésions anatomiques :

- Une rupture de l'albuginée a été traitée par suture après résection de la pulpe extériorisée dans 37 cas (Figure 1 et 2).

- Une fracture testiculaire complexe ayant nécessité une orchidectomie dans 8 cas.

- Un hématome intra testiculaire a été évacué par incision de l'albuginée dans 10 cas et respecté dans 2 cas vue son caractère non compressif (Figure 3).

- Une hématocèle a été traitée par drainage chirurgical dans 50 cas.

- Un seul cas de luxation testiculaire inguinale a été observé et a nécessité une orchidectomie.

- Une torsion post traumatique du cordon spermatique a été observée dans 7 cas et traitée par détorsion et orchidopexie (2 cas) ou orchidectomie (5 cas).

- Les contusions épididymaires ont été respectées dans 7 cas, de même qu'une désinsertion épididymaire partielle. Par contre une plastie épididymaire a été pratiquée dans 4 cas.

- Un hématome du cordon spermatique a été respecté dans 2 cas et drainé chirurgicalement dans 4 cas.

II n'a pas été mis en évidence de différence significative en terme de taux d'orchidectomie initiale en fonction

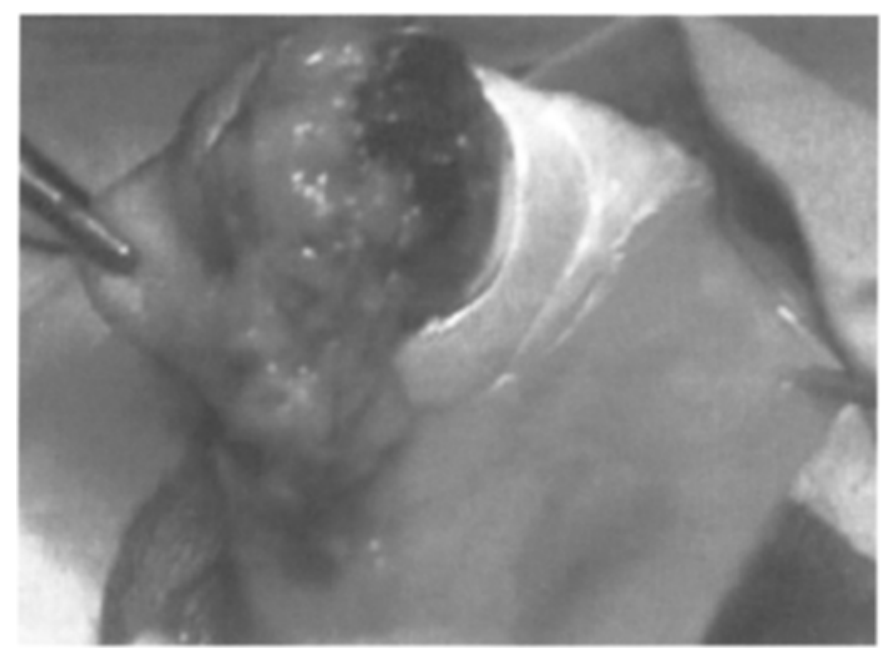

Figure 1 : Aspect peropératoire d'une fracture testiculaire.

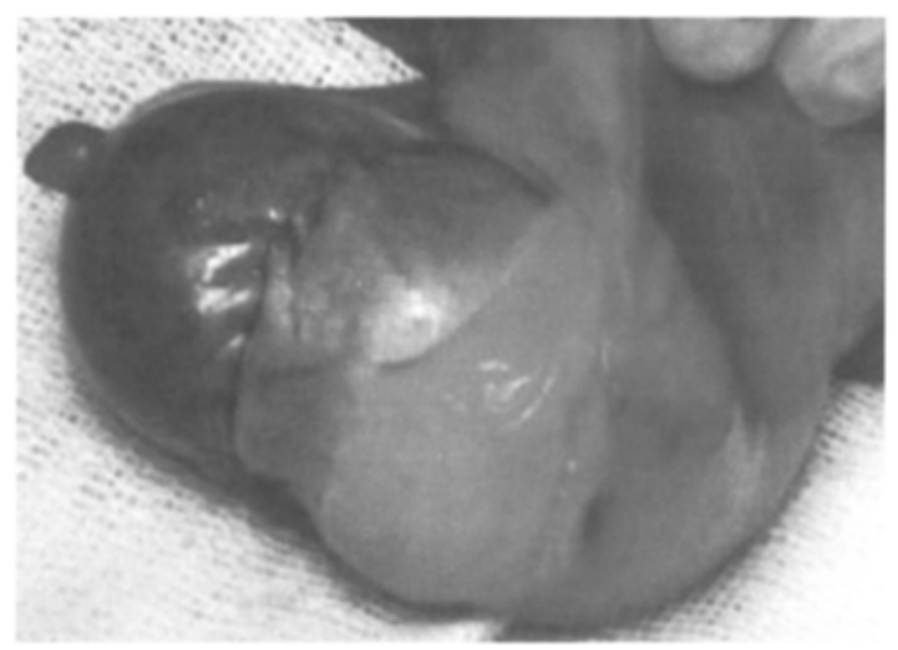

Figure 2 : Fracture testiculaire après suture de l'albuginée.

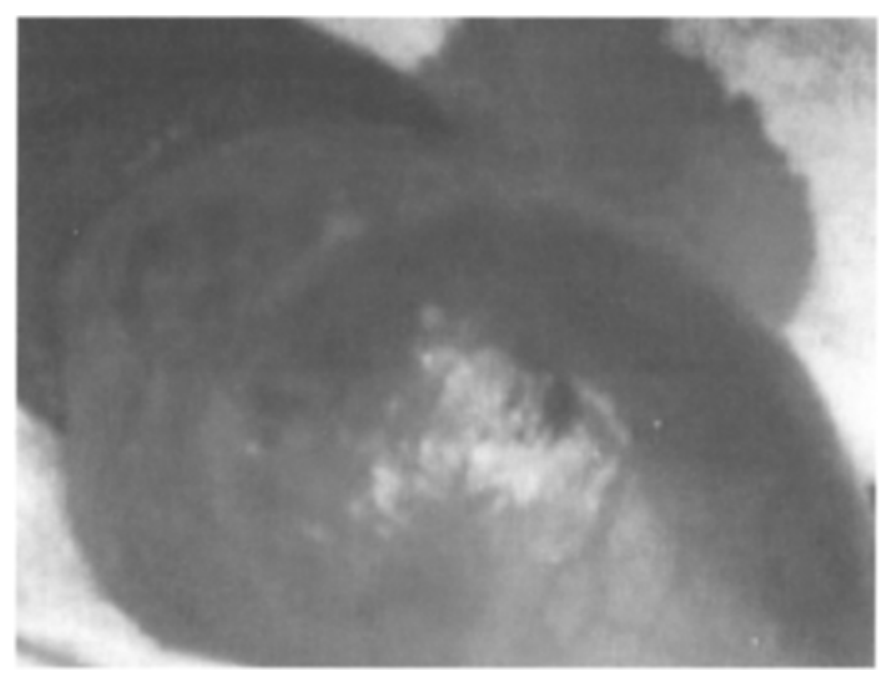

Figure 3 : Aspect peropératoire d'un hématocèle. 
du délai d'intervention, avec $13,9 \%$ pour les patients opérés dans les trois premiers jours et $13,8 \%$ après ce délai. La durée moyenne de l'hospitalisation était de 2 jours avec des extrêmes allant de 1 à 10 jours.

L'évolution a pu être appréciée chez 72 patients, traités chirurgicalement (59 cas) et médicalement (13 cas). Vingt neuf patients $(40,2 \%)$ ont présenté des complications dont 24 avaient été traités chirurgicalement soit $82,7 \%$ des cas. Une suppuration pariétale a été observée chez 6 patients $(10,1 \%)$ traités chirurgicalement avec évolution favorable sous soins locaux.

L'évolution à distance a été appréciée avec un recul moyen de 8 mois (Tableau 3 ). Treize patients (18\%) ont gardé des douleurs testiculaires résiduelles. Une atrophie testiculaire a été observée dans 4 cas $(5,5 \%)$ et une hydrocèle dans 3 cas $(4,1 \%)$. Par ailleurs un patient a présenté une dysfonction érectile d'origine vraisemblablement psychogène. De même un cas de dépression a été observé (Tableau 3).

L'étude du sperme n'a été réalisée que chez un seul patient traité chirurgicalement pour fracture testiculaire et ayant eu un traitement conservateur. Le spermogramme pratiqué 12 mois après le traumatisme a mis en évidence une asthénospermie ( $40 \%$ de formes mobiles) et une vitalité assez diminuée (44\%). La recherche d'anticorps anti-spermatozoïdes était négative.

\section{DISCUSSION}

Les traumatismes des bourses constituent un accident relativement peu fréquent par rapport aux autres pathologies traumatiques de l'appareil urinaire de l'adulte jeune $[2,3,22]$. Le nombre moyen rapporté dans les différentes séries varie de 3,3 à 5,6 cas/an [2, 3, 14]. Dans notre série, il est de l'ordre de 9,3 cas par an. L'attitude préconisée dans le service est d'hospitaliser tout traumatisé des bourses afin de ne pas méconnaître une lésion testiculaire pouvant avoir de graves conséquences et de prévenir des problèmes médicolégaux qui pourraient s'ensuivre.
Ces traumatismes affectent plus fréquemment l'adolescent et l'adulte jeune $[2,4,5,12,14]$. Le délai entre le traumatisme et la consultation est souvent important. Le délai moyen dans notre série est de 18,5 jours. Mac Dermott et al. [18] estiment que la sédation assez rapide de la douleur après le choc peut être responsable de ce retard de consultation. Ce délai pourrait aussi s'expliquer par le nombre élevé de traumatisme d'origine criminelle et par le jeune âge de la population souvent peu compliante et peu encline à consulter [14].

Les rixes et les agressions représentent l'étiologie la plus fréquente dans notre série $(35,1 \%)$, ce qui est le cas dans plusieurs séries $[3,11,12,16,23]$. Le testicule est protégé par sa situation anatomique, sa mobilité dans la bourse et par la solidité de l'albuginée [2, 14]. En effet, seul un traumatisme violent périnéal par choc direct dirigé de bas en haut et légèrement en dedans peut entraîner une lésion testiculaire $[4,9,12]$.

La symptomatologie clinique dépend de l'importance du choc, des lésions anatomiques et du délai de la première consultation $[1,9,20]$. Le tableau clinique est en règle dominé par la douleur scrotale $(73,8 \%)$ et l'augmentation du volume de la bourse $(89,2 \%)$ ce qui peut rendre l'examen clinique difficile voir impossible. Ainsi, l'hématocèle, facteur de gravité traduisant en général une rupture du testicule, n'a pu être retrouvé à l'examen clinique que dans 3 cas. La faible sensibilité de ce signe clinique, $5,6 \%$ dans notre série et $11 \%$ pour Kleinclauss et al. [14], rend le diagnostic du traumatisme grave du testicule difficile à poser cliniquement.

Ainsi le seul recours à l'interrogatoire et à l'examen clinique parait généralement insuffisant pour établir un bilan lésionnel précis et complet, ce qui incite de nombreux auteurs à proposer une exploration chirurgicale devant un tableau comportant une douleur et une augmentation de volume de la bourse [2]. Cette attitude a été quelque peu modifiée par les progrès de l'échographie testiculaire.

Tableau 3 : Complications tardives des traumatismes testiculaires (72 patients).

\begin{tabular}{|c|c|c|c|}
\hline Séquelles & $\begin{array}{c}\text { Traitement médical } \\
13 \text { cas }(18 \%)\end{array}$ & $\begin{array}{c}\text { Traitement chirurgical } \\
59 \text { cas }(82 \%)\end{array}$ & $\begin{array}{c}\text { Ensemble des patients } \\
72 \text { cas }(100 \%)\end{array}$ \\
\hline Atrophie & $1(7,6 \%)$ & $3(5 \%)$ & $4(5,5 \%)$ \\
\hline Douleurs résiduelles & $1(7,6 \%)$ & $12(20,3 \%)$ & $13(18 \%)$ \\
\hline $\begin{array}{l}\text { Dysfonction érectile } \\
\text { Dépression }\end{array}$ & $\begin{array}{l}1(7,6 \%) \\
1(7,6 \%)\end{array}$ & $\begin{array}{l}0 \\
0\end{array}$ & $\begin{array}{l}1(1,4 \%) \\
1(1,4 \%)\end{array}$ \\
\hline Hydrocèle & $1(7,6 \%)$ & $2(3,3 \%)$ & $3(4,1 \%)$ \\
\hline Pas de séquelles & $8(61,5 \%)$ & $42(71,1 \%)$ & $50(69,4 \%)$ \\
\hline
\end{tabular}


La place de l'échographie reste controversée dans la prise en charge en urgence des traumatismes fermés des bourses. Elle doit être réalisée par un opérateur habitué à cette pathologie avec recours à des sondes de haute fréquence $(7,5$ à $10 \mathrm{MHz})$. Elle permet la réalisation d'un bilan lésionnel assez précis en montrant une éventuelle rupture de l'albuginée, ce qui va conforter l'indication opératoire. En effet, certains auteurs [2, 6, $13]$ lui trouvent une bonne sensibilité ( $94 \%$ à $100 \%)$ pour le diagnostic de rupture de l'albuginée. Ces chiffres sont cependant contestés par Cass et Luxenberg [7] et Kleinclauss et al. [14] qui retrouvent une sensibilité respective de $28 \%$ et $58 \%$. C'est le cas de notre série où la sensibilité de l'échographie pour la détection d'une rupture de l'albuginé n'est que $34,7 \%$ avec 13 faux négatifs.

Par ailleurs, l'appréciation des autres lésions du contenu intra scrotal reste variable. En effet plusieurs auteurs ont rapporté une faible sensibilité de l'échographie dans la différenciation entre une contusion et une fracture testiculaire [7], d'autres ont démontré que l'hématocèle et l'hématome intra testiculaire sont bien vus par cet examen [20].

L'échographie a une sensibilité de $75 \%$ pour la détection de l'hématocèle [14], ce qui concorde avec les résultats de notre série $(76,9 \%)$. De plus, nous avons retrouvé une bonne sensibilité pour le diagnostic des contusions testiculaires (100\%), alors qu'elle est de $33,3 \%$ pour la détection des hématomes intra-testiculaires.

Il apparaît donc que l'échographie peut aider le clinicien dans la réalisation d'un bilan sommaire des lésions, mais elle ne doit pas dispenser d'une exploration chirurgicale en cas de doute clinique [14]. Ainsi, à notre sens, l'échographie ne doit pas retarder l'exploration chirurgicale lorsque celle-ci est d'emblée justifiée par l'examen clinique. En revanche lorsque l'examen clinique parait peu alarmant, l'échographie permet de vérifier l'intégrité du testicule et d'envisager pour ces traumatismes considérés comme bénins un traitement médical [8].

Par ailleurs, selon certains auteurs, I'IRM paraît être de meilleure sensibilité que l'échographie pour le diagnostic des petites déchirures de l'albuginée et non visibles à l'échographie, mais cette exploration est rarement accessible en urgence [21, 10].

Plusieurs auteurs ont prouvé que l'exploration précoce de la bourse traumatisée réduirait la morbidité et le taux d'orchidectomie. Par contre, Kleinclauss et al. [14] ne trouvent pas de différence significative, en terme de taux d'orchidecomie et de taux de séquelles à distance, en fonction du délai d'intervention. Ceci est le cas de notre série.
L'évolution à long terme des traumatismes testiculaires pourrait être émaillée par la survenue de complications

- L'atrophie testiculaire est assez fréquente et pourrait atteindre $50 \%$ des patients $[4,18,19]$. Cette complication a été observée dans $5,5 \%$ des cas de notre série. Certains mécanismes ont été évoqué dans la genèse de l'atrophie testiculaire : des lésions post-traumatiques de la micro-vascularisation du testicule, une ischémie par compression de la pulpe testiculaire contre l'albuginée par l'oedème et ou par I'hématome, des mécanismes auto-immuns [8]. Par ailleurs des cas d'atrophie du testicule controlatéral au traumatisme ont été décrits [8].

- Les douleurs testiculaires résiduelles surviennent dans environ $10 \%$ des cas $[14,15]$. Cass et Luxenberg [7] supposent que l'exploration chirurgicale permet de diminuer les douleurs par l'évacuation de l'hématome scrotal. Dans notre étude, la tendance est plutôt inverse, avec $20,33 \%$ de douleurs résiduelles après traitement chirurgicale contre $7,6 \%$ après traitement médical seul, ce qui rejoint les résultats de Kleinclauss et al. [14].

- Les troubles de la fertilité, dont le risque est estimé à $5 \%$ [14], sont liés à l'apparition d'anticorps antispermatozoïdes secondaires à l'effraction de l'albuginée [25]. Ces anticorps ne sont probablement pas le seul facteur expliquant l'infertilité. En effet, Kunadia et al. [15], dans une série de huit patients infertiles suite à un traumatisme testiculaire, ont retrouvé des anticorps sériques chez un seul patient. Shaul et al. [24] ont démontré chez le rat que la rupture de l'albuginée ne constituait pas un facteur de risque d'infertilité.

Lin et al. [17] ont montré, dans une étude de dix traumatismes testiculaires, que le spermogramme des patients ayant eu un traitement chirurgical conservateur (résection de la pulpe extériorisée et suture de l'albuginée) n'était pas différent d'un groupe témoin, et que seule l'orchidectomie modifiait la densité du sperme.

\section{CONCLUSION}

La prise en charge précoce et adéquate de tout traumatisme testiculaire reste le seul garant pour la sauvegarde du testicule et de ses fonctions. Ainsi nous proposons un arbre décisionnel devant un traumatisme fermé du scrotum :

- devant une grosse bourse avec testicule non palpable, l'exploration chirurgicale en urgence devrait être systématique ;

- devant une grosse bourse avec testicule palpable et/ou un doute clinique sur l'intégrité du contenu 
scrotal, l'échographie doit être demandée sans retarder un éventuel geste chirurgical. Ainsi le traitement médical n'est indiqué que devant un hématome pariétal, une contusion testiculaire ou épididymaire ;

- devant la persistance d'un doute à l'échographie sur l'intégrité du contenu scrotal, l'exploration chirurgicale doit être réalisée afin de faire un bilan lésionnel précis et d'entreprendre une attitude chirurgicale adéquate.

Le suivi des patients traumatisés doit être amélioré afin de dépister et de prendre en charge les problèmes de fertilité.

\section{REFERENCES}

1. ALBERT N.E. : Testicular ultrasound for trauma. J. Urol., 1980, $124: 558-559$.

2. ALTARAC S. : Management of 53 cases of testicular trauma. Eur. Urol., 1994, $25: 119-123$.

3. BARTHELEMY Y., DELMAS V., VILLERS A., BARON J.C., SIBERT A., BOCCON-GIBOD L. : Traumatismes des bourses: à propos de 33 cas. Prog. Urol., 1992, 2 : 628-634.

4. BENCHEKROUN A., IKEN A., KASMAOUI E. et al. : Traumatismes des bourses : à propos de 40 cas. Ann. Urol. (Paris), 2001, $35:$ 349-352.

5. BENCHEKROUN A., LAKRISSA A., ABAKKAT T. : Les contusions des bourses et de leur contenu (à propos de 30 cas). Marroc Med., 1985, $7: 668-673$.

6. BUCKLEY J.C., MCANINCH J.W. : Use of ultrasonography for the diagnosis of testicular injuries in blunt scrotal trauma. J. Urol., 2006, $175: 175-178$.

7. CASS A.S., LUXENBERG M. : Value of early operation in blunt testicular contusion with hematocele. J. Urol., 1988, 139 : 746-747.

8. CULTY T., RAVERY V. : Traumatismes scrotaux : stratégie de prise en charge. Ann. Urol., 2006, 40:117-125.

9. DESCOTES J.L., COQUILHAT P., MICHELA., RAMBEAUD J.J. : Traumatisme des bourses. Encycl. Med. Chir. (Paris) Néphrol. Urol., 1995, 18.625.A.10.

10. DESCOTES J.L., HUBERT J. : Traumatismes des testicules et de la verge. Prog. Urol., 2003, $13:$ 1157-1160.

11. ELHADAD A., DEBESSE B. : Contusions des bourses et leurs contenu (à propos de 13 cas opérés). Ann. Chir., 1977, $31: 455-461$.

12. EL MOUSSAOUI A., JOUALE A., BENJELLOUN S. Traumatismes des bourses. J. Urol. (Paris), 1996, 102 : 8891.

13. FOURNIER G.R. JR., LAING F.C., JEFFREY R.B., MCANINCH J.W. : High resolution scrotal ultrasonography: a highly sensitive but non specific diagnostic technique. J. Urol., 1985, 134 : 490-493.
14. KLEINCLAUSS F., MARTIN M., CHABANNES E., BERNARDINI S., DELLA NEGRA E., BITTARD $H$. : Traumatismes testiculaires : à propos de 56 cas. Prog. Urol., 2001, $11: 486-491$.

15. KUNADIAA.K., ERCOLE C.J., GLEICH P., HENSLEIGH H., PRIOR J.L. : Testicular trauma : potential impact on reproductive function. J. Urol., 1996, 156 : 1643-1646.

16. LEWIS C.A., MICHELL M.J. : The use of real-time ultrasound in the management of scrotal trauma. Br. J. Radiol., 1991, 64: 792-795.

17. LIN W.W., KIM E.D., QUESADA E.T., LIPSHULTZ L.I., COBURN M. : Unilateral testicular injury from external trauma: evaluation of semen quality and endocrine parameters. $\mathrm{J}$. Urol., 1998, $159: 841-843$.

18. MCDERMOTT J.P., GRAY B.K., HAMILTON STEWART P.A.: Traumatic rupture of the testis. Br. J. Urol., 1988, 62 : 179181.

19. MCDERMOTT J.P., GRAY B.K. : Bilateral testicular atrophy following blunt trauma. Br. J. Urol., 1989, 63 : 215-216.

20. MARTINEZ-PINEIRO L., CELERO E., COZAR J.M., AVELLANA J.A., MORENO J.A., MARTINEZ-PINEIRO J.A.: Value of testicular ultrasound in the evaluation of blunt scrotal trauma without haematocele. Br.J.Urol., 1992, 69 : 286-290.

21. MUGLIA V, TUCCI S., ELIAS J., TRAD C.S., BILBEY J., COOPERBERG P.L. : Magnetic resonance imaging of scrotal diseases: when it makes the difference. Urology, 2002, 59 : 419-423.

22. MULHALL J.P., GABRAM S.G., JACOBS L.M. : Emergency management of blunt testicular trauma. Acad. Emerg. Med., $1995,2: 639-643$.

23. SELLEM G., TOBELEM G., ECONOMOU C., AMER M., ARVIS G. : Intérêt de l'échographie dans les contusions des bourses. Ann. Urol., 1987, $25: 327-330$.

24. SHAUL D.B., XIE H.W., DIAZ J.F., MAHNOVSKI V., HARDY B.E. : Surgical treatment of testicular trauma : effects on fertility and testicular histology. J. Ped. Surg., 1997, 32 : 8487.

25. SRINIVAS M., HASHIM S., MITRA DK. : Unilateral blunt testicular trauma in per-pubertal rats. Pediatr. Surg. Int., 1999, $15: 457-460$.

Manuscrit reçu : mai 2006 ; accepté octobre 2006. 


\section{ABSTRACT}

Blunt scrotal trauma: management strategy

Hammadi FAKHFAKH, Kamel CHABCHOUB, Abdelkader BOUHLEL, Hafedh KETATA, Hatem ALLOUCH, Ali BAHLOUL, Mohamed Nabil MHIRI

Introduction: Blunt scrotal trauma is increasingly frequent, but surgical exploration of these cases of trauma remains controversial. The objectives of this study were to assess the diagnostic value of clinical examination and ultrasound in testicular trauma and to analyse the complications of the various treatments proposed (surgical and medical treatments), in order to more clearly define the place of medical or surgical treatment in this form of trauma in young adults.

Patients and Methods: 130 cases of blunt scrotal trauma were managed between 1993 and 2004. In the absence of clinical and ultrasound criteria of severity (haematocele, very large intratesticular haematoma, rupture of the tunica albuginea), medical treatment consisting of rest, antiinflammatory drugs, and testicular support was instituted. Surgical exploration was performed when serious lesions of the testis were suspected. Scrotal ultrasound was performed in 68 patients and 46 of them underwent scrotal exploration. The sensitivity and specificity of scrotal ultrasound were determined by comparing radiological findings with definitive intraoperative findings. The immediate morbidity and long-term sequelae were analysed.

Results: The mean age of the patients was 24 years (range: 4 to 73 years). The clinical features were dominated by pain $(73.8 \%)$ and scrotal swelling $(89.2 \%)$. The sensitivity of testicular ultrasound was $\mathbf{3 4 . 7 \%}$ for testicular rupture, $100 \%$ for testicular contusions, $33.3 \%$ for intratesticular hematoma and $76.9 \%$ for haematocele.

Medical and surgical treatments were instituted in $\mathbf{2 9 . 2} \%$ and $70.7 \%$ of cases, respectively. With a mean follow-up of 8 months, chronic pain and testicular atrophy were observed in $18 \%$ and $5.5 \%$ of cases, respectively.

Conclusion: In the absence of signs of severity, medical treatment with regular surveillance remains justified. However, in the presence of doubtful clinical or ultrasound findings, surgical exploration must be performed as soon as possible.

Key words : trauma, scrotum, testis, ultrasound. 